\title{
Percepciones de futuros docentes sobre el uso de tecnología en educación inicial ${ }^{1}$
}

\section{Perceptions of Future Teachers on the Use of Technology in Early Childhood Education}

Tatiana Ghitis Jaramillo $\left(^{*}\right)$ tatianaghija@unisabana.edu.co
Alexander Alba Vásquez $(*)$ John.alba@unisabana.edu.co

(1) Universidad de La Sabana

(Recibido: 8 de junio de 2017; Aceptado para su publicación: 6 de noviembre de 2017)

Cómo citar: Ghitis, T. y Alba, A. (2019). Percepciones de futuros docentes sobre el uso de tecnología en educación inicial. Revista Electrónica de Investigación Educativa, 21, e23, 1-12. doi:10.24320/redie.2019.21.e23.2034

\section{Resumen}

El propósito de este artículo es dar a conocer la percepción de futuros educadores sobre la necesidad, uso e importancia de implementación y la formación requerida para una práctica pedagógica que incluya las herramientas tecnológicas como mediaciones didácticas. La investigación se realizó bajo un enfoque cualitativo con alcance descriptivo y diseño transeccional que incluye recolección de datos cuantitativos. Los instrumentos de recolección de información fueron un cuestionario y grupos focales. La población se conformó por 175 estudiantes de licenciatura en educación inicial, con un muestreo aleatorio estratificado de 100 estudiantes. El principal hallazgo indica que aunque los estudiantes encuestados pertenecen a la generación millenial y son nativos digitales, al reflexionar sobre su práctica profesional no ven un uso potencial de la tecnología como herramienta didáctica, lo que puede considerarse como un obstáculo para la enseñanza.

Palabras clave: Tecnología educacional, formación profesional superior, educación de la primera infancia, percepción social.

\section{Abstract}

The purpose of this article is to present future educators' perception of the need for, use of, and importance of implementing ICT, and the training required for teaching practices that use technological tools. The study followed a qualitative approach, and is descriptive in scope with a cross-sectional design that includes quantitative data collection. Information was collected through a questionnaire and focus groups. The population comprised 175 undergraduate students of early childhood education, with a stratified random sample of 100 students. The main finding is that although the students surveyed are digital natives from the Millennial generation, when reflecting on their professional practice they did not see technology as having potential as an educational tool, which may be considered an obstacle for teaching.

Keywords: Educational Technology, professional training, early childhood education, social perception.

\footnotetext{
${ }^{1}$ Universidad de La Sabana, proyecto Posibilidades didácticas del uso de robots humanoides en educación inicial EDU $-37-2015$
} 


\section{Introducción}

La cotidianidad se encuentra permeada por diversas herramientas y artefactos tecnológicos que la apoyan. Estos elementos se han convertido en un soporte necesario que apoya procesos comunicativos, médicos, industriales, laborales y educativos. Desde el siglo pasado, las computadoras ingresaron a las instituciones educativas y los expertos buscaban capacitar a los estudiantes en su manejo, como parte de las competencias tecnológicas que era necesario desarrollar en los procesos educativos iniciales. Pero sólo hasta el siglo XXI la mayoría de instituciones educativas pudieron acceder a estos elementos tecnológicos con el fin de disminuir las brechas sociales causadas por la poca posibilidad de acceso.

En las instituciones educativas se comenzó a promover la inclusión de la tecnología por medio de aulas con elementos electrónicos como computadoras portátiles, tabletas, tableros digitales y smartphones; pero, en la mayoría de los casos no se han analizado las competencias tecnológicas y didácticas que el docente de preescolar requiere para hacer un uso apropiado de estos elementos.

La UNESCO (2008) expone que existe una necesidad obligatoria de aprender a utilizar y a enseñar la tecnología para resolver problemas de la vida cotidiana. Plantea tres enfoques relacionados con las competencias en TIC que requiere un docente para implementar, de manera apropiada, la tecnología en su salón de clases, estos son: nociones básicas de tecnología, profundización de los conocimientos y creación de conocimientos (p. 5).

El primer enfoque busca que el docente incluya, de alguna manera, las competencias tecnológicas en su trabajo de aula por medio del manejo de herramientas básicas y la alfabetización digital; el segundo enfoque fomenta la resolución de problemas reales por medio de los conocimientos adquiridos que mejoren los procesos económicos y sociales de los ciudadanos, el docente se convierte en guía y busca que sus estudiantes apliquen el conocimiento adquirido por medio de herramientas tecnológicas complejas y el trabajo en grupos colaborativos; el tercer enfoque exige el desarrollo de las competencias y habilidades para el siglo XXI, requiere que los estudiantes logren autogestionar sus diseños у сгеar, producir y manejar todo tipo de tecnología, por medio de organizaciones de aprendizaje en las que el docente es un modelo a seguir.

Estos enfoques se convierten en lineamientos de las universidades formadoras de docentes, pero... ¿qué piensan sobre la inclusión de tecnología en el aula de preescolar los profesores en formación?

La presente investigación se centra en las percepciones de un grupo de docentes en formación con respecto al uso de la tecnología como herramienta didáctica que apoya los procesos de enseñanza y aprendizaje. Sus percepciones se vuelven prioritarias para lograr su inclusión adecuada en el aula, pues al indagar sobre su uso, relevancia, necesidad y rol facilitador, se comprenderá la perspectiva de organización y planeación de actividades que los futuros docentes realizarán y que hará evidente la inclusión "amigable y adecuada" que apoye al docente y motive al estudiante desde una perspectiva real de aporte pedagógico.

\subsection{Antecedentes}

Investigaciones relacionadas con las percepciones y creencias, uso y aplicaciones de la tecnología en el aula por parte de los docentes fueron determinantes para este estudio. En las investigaciones centradas en los profesores se demuestra la relevancia de las percepciones docentes en cuanto a las potencialidades de la tecnología para el aprendizaje de los alumnos, enmarcándose en qué se puede hacer y en cómo se pueden implementar (Núñez, Conde, Ávila y Mirabent, 2015).

Es importante resaltar el estudio de Cabero (2016), en el que se hace un análisis sobre el camino investigativo centrado en tecnología educativa y cómo este camino da una perspectiva para abordar investigaciones futuras. Dentro de los elementos que cabe resaltar se encuentran: la necesidad de transferir procesos tecnológicos que se adapten al contexto, con el fin de resolver problemas propios de la realidad en la que se desenvuelve el estudiante; la problemática actual del crecimiento desmedido de la investigación superflua y poco profunda en este eje.

Cabero (2016) expone algunas conclusiones: la necesidad de ampliar la fundamentación teórica en este campo para las investigaciones, la superación de la comparación de diversos medios, fundamentándose en la curva de atención que suele tener un estudiante al interactuar con una innovación dentro del aula; la 
ampliación de los sujetos de estudio o los informantes en el proceso investigativo, pues se evidencia una necesidad en la contextualización del conocimiento adquirido, así como la inclusión de los docentes y sus percepciones, y se fomenta el análisis de los resultados más allá de la retención de información, teniendo en cuenta las demás dimensiones (motriz, cognitiva, comunicativa, socio-afectiva) y el desarrollo de procesos cognitivos de orden superior.

La construcción y opinión del profesor, encargado de implementar la tecnología en el aula, se convierte en un elemento primordial en la creación de proyectos educativos que incluyan elementos tecnológicos, ya que de su aceptación depende el ingreso al aula.

Se resalta la importancia de enseñar a los docentes no sólo a utilizar la tecnología sino a incluirla en sus planes de estudio e implementarla en sus clases con el objetivo de que sus estudiantes realicen procesos cognitivos complejos (Mundy, Kupczynski y Kee, 2012) pues, la mayoría de docentes utiliza la tecnología para actividades administrativas o para el envío de instrucciones (Morales, Trujillo y Raso, 2015). A pesar de sentirse preparados para utilizarla y mostrar una visión positiva frente a su uso, dan a conocer la necesidad de formación y capacitación en su adecuada implementación (Dorfsman, 2012; Tello y Cascales, 2015).

Queda demostrada la necesidad de capacitación y la posibilidad de investigación sobre las percepciones de los docentes de educación inicial, ya que los trabajos revisados se centran en educación básica primaria, secundaria y superior.

\subsection{Acercamientos teóricos}

Los principales términos en los que se enmarcó el proyecto fueron: Tecnología y percepciones.

Tecnología. La tecnología ha permeado la vida del hombre al hacerse indispensable para su desarrollo personal. Elementos de la vida cotidiana como el refrigerador, el televisor, el celular y la computadora, son herramientas que facilitan el quehacer cotidiano de las personas, mejorando su calidad de vida y permitiendo economizar tiempo y recursos.

A partir del primer computador, creado por Turing (1950), la sociedad de científicos ha modificado ese aparato para convertirlo en un elemento portátil al: disminuir su tamaño, mejorar su rapidez de procesamiento e incluir software y aplicaciones de optimización que facilitan el manejo y el acceso a la información. Lo que antes era un lujo para las personas, ahora se ha convertido en una necesidad para todos los profesionales y estudiantes, porque apoya la búsqueda, acceso y procesamiento de información y la organización del tiempo; genera cambios en el estilo de vida al permitir la protección de las casas e instituciones, ayuda a salvar vidas en disciplinas como la Medicina al tratar diversas enfermedades (Bustos, Aguas, Díaz y Roa-Guerrero, 2017; Kashiwagi et al., 2014; Mulligan, Rowland, Sandlund, Potterton y Kanagasabai, 2015).

Se habla de artefactos específicos para el uso educativo; en Robots for kids, Druin y Hendler (2000) dieron a conocer 15 años de evolución de la tecnología y la robótica en el aspecto educativo, en el libro exponen la interacción que se crea entre los artefactos tecnológicos y los niños, con el fin de generar mayor motivación por el aprendizaje, esto se transforma en un punto de partida que conlleva a demostrar la importancia de la implementación de tecnología en el aula.

Sobre esta generación habló Prensky (2001), refiriéndose a los nativos digitales -término que atribuye a aquellos niños y adolescentes nacidos en el siglo XXI acostumbrados a usar la tecnología de forma intuitiva, pues se les facilita aprender de ella. Estos términos son controversiales para autores como Burin, Coccimiglio, González y Bulla (2016) y Escofet, López y Alvarez (2014), quienes no hablan de una natividad sino de una naturalidad para el aprendizaje y uso de estos artefactos tecnológicos en ámbitos no necesariamente académicos.

Actualmente se han creado nuevos términos para clasificar a estas generaciones, como Millenial, Y y $Z$ (TED, 2011; Piscitelli, 2009; Shatto y Erwin, 2016), generación @ o Red (Ortiz, 2011) o generación Multimedia (Morduchowicz, 2012), pues la evolución tecnológica ha evidenciado cambios generacionales que se dan a conocer en las instituciones entre los docentes y los estudiantes, permitiendo que se generen nuevos procesos de aprendizaje y exploración del medio. 
La Organización para la Cooperación Económica y el Desarrollo (OECD, 2002) define las TIC como "aquellos dispositivos que capturan, transmiten y despliegan datos e información electrónica y que apoyan el crecimiento y desarrollo económico de la industria manufacturera y de servicios" (p. 2). Los beneficios de su uso en el ámbito educativo son evidentes.

Tecnologías para el Aprendizaje y el conocimiento. La educación no se queda atrás, actualmente se habla de la implementación educativa de las TIC en el aula como herramientas poderosas de aprendizaje, llamadas actualmente TAC (Tecnologías para el Aprendizaje y el Conocimiento) por su aporte al campo educativo, dentro de las cuales se incluye el uso de la computadora, la tableta, el celular e incluso los robots; sin embargo, los docentes parecen no estar preparados para esta inclusión, pues se evidencia formación, pero muy poco cambio (Fernández-Cruz y Fernández-Díaz, 2016) y los estudiantes los utilizan más como medios de entretenimiento que como aprendizaje (Sánchez-Labella, 2015). Pero la solución está en capacitar a los docentes en la creación de ambientes de aprendizaje computarizados con el fin de fomentar condiciones adecuadas para que los procesos de enseñanza sean apropiados y los de aprendizaje sean optimizados (Guacaneme-Mahecha, Gómez-Zermeño y Zambrano-Izquierdo (2016); Roblizo y Cózar, 2015).

Dentro de los ambientes, la interacción con los artefactos tecnológicos se convierte en una característica fundamental para el éxito, mientras se permita una flexibilidad de uso que atienda a los diversos estilos de aprendizaje y a las particularidades de la población infantil con la que se está trabajando. Actualmente existe el término aprendizaje "STEAM" (ciencia, tecnología, ingeniería, arte y matemática) que aporta a mejorar las habilidades del siglo XXI desde la educación inicial, y facilita la construcción interdisciplinar de aprendizajes en la solución colaborativa de problemas (Ok, Cho y Cho, 2016), y promueve la integración de disciplinas consideradas incompatibles en épocas anteriores, como la matemática y el arte, al desarrollar procesos metacognitivos útiles para los retos actuales (McAuliffe, 2016).

Galvis (2004) expone cuatro formas diferentes para trabajar en los ambientes mediados por tecnología, el primero se refiere al uso en un entorno virtual de aprendizaje, ya que apoya el proceso de formación de estudiantes por medio del uso de páginas web y tutoriales; el segundo incluye la tecnología dentro del aula por medio de la experimentación de diversos objetos de estudio a través de los aparatos electrónicos, como calculadoras, simuladores, herramientas de productividad, entre otros; el tercero se refiere al aprendizaje colaborativo mediado por plataformas virtuales que incluyen chat, foros, juegos y videoconferencias; y por último, la simulación para el aprendizaje de habilidades prácticas.

Litwin (2005), complementa estas afirmaciones con una fuerte crítica:

Cincuenta años después, las nuevas tecnologías son incorporadas por las mismas razones en las mismas escuelas. Por su simple introducción se convierten, casi por obra de un pensamiento mágico, en el camino más directo y efectivo para alcanzar la resolución de todos los problemas de la educación (p. 23).

No se trata sólo de introducir la tecnología en el aula, sino de hacerlo de forma adecuada y con objetivos pedagógicos claros. Parte de la dificultad se encuentra relacionada con la cultura didáctica disciplinar, que demuestra costumbres establecidas a lo largo del tiempo por parte de los docentes en el uso de recursos y sus formas de enseñar (Pozuelo, 2014), en muchos casos esa cultura arraigada se complementa con un miedo o desconfianza por el uso de las Tic en el aula, argumentando que las metodologías y técnicas que se han utilizado por mucho tiempo son confiables.

TAC en preescolar. Como se observa, el ingreso de la tecnología al aula ha sido largo y complejo, y más aún si se habla de aulas de educación inicial, donde el uso de las TAC se limita a las computadoras, grabadoras y televisores (Litwin, 2005), y sólo el docente las puede utilizar por miedo a que los aparatos sean dañados por los niños a causa de su desconocimiento en el manejo, a pesar de que en la población colombiana (que incluye niños mayores de 5 años), el 49.1\% tiene acceso a computadoras, tabletas y smartphones, y el $45.8 \%$ cuenta con Internet en sus casas (DANE, 2017), aunque el uso que se le da es diferente en cada espacio.

La implementación de las TAC en el aula de preescolar obliga a un cambio de paradigmas propios de los directivos y los docentes, que permita una modificación curricular que facilite incluir en el trabajo experiencial la interacción con tableros inteligentes, materiales educativos computarizados y objetos 
virtuales de aprendizaje (Marques, 2013). Que, además de apoyar al docente en el apoyo a estudiantes con algún tipo de dificultad o problemática que no le permita desenvolverse de forma adecuada en sus procesos educativos (Drigas, Kokkalia y Lytras, 2015), puedan empoderar a los niños en un manejo adecuado y crítico de estos elementos.

Se reconoce que existen beneficios para los estudiantes de preescolar cuando se exponen a la tecnología en edades tempranas, al contribuir de forma positiva a su aprendizaje y desarrollo (Liu, Toki y Pange, 2014). Aunque, por otro lado, hay quienes rechazan este postulado, por ir en contra de la posibilidad de exploración y construcción independiente del aprendizaje por medio del juego, en su concepción tradicional, que permite al niño actuar de forma activa y movilizarse en su entorno (Lindahl y Folkesson, 2012).

\subsection{La percepción como proceso de construcción de conocimiento}

A continuación se presentan algunas definiciones sobre percepción relacionadas con la postura de la investigación.

Neisser (1981) se refiere a la percepción como "un proceso activo-constructivo en el que el perceptor, antes de procesar la nueva información, y con los datos archivados en su conciencia, construye un esquema informativo anticipatorio", dicho esquema tiene en cuenta una "imagen mental que se forma con ayuda de la experiencia y necesidades. Es resultado de un proceso de selección, interpretación y corrección de sensaciones" (Guardiola, 2014, p. 1), propias de cada individuo.

Por otro lado, el proceso perceptivo "no es una copia de la realidad, sino un proceso constructivo mediante el que se interpretan datos sensoriales" (Neisser, 1981, como se citó en Carbajo y Carcelén, 2016, p. 130), las percepciones son particulares y se fundamentan en conocimientos y experiencias previas. Tienen tres características: son subjetivas, porque dependen de la persona; selectivas, porque el cerebro elige los elementos que generan deseos e intereses a partir de lo que se siente y se percibe; y temporales, porque es posible que esas percepciones cambien con nuevas experiencias o situaciones (Vergara, 2012).

Según Neisser (1981), es importante tener en cuenta que existen dos componentes principales presentes en la percepción: las sensaciones y los inputs internos, estos últimos compuestos por tres factores: la necesidad, la motivación y la experiencia, sin los cuales el proceso de percepción estaría incompleto. Cada uno de los inputs ayuda a que las percepciones sean subjetivas y cambien entre un individuo y otro.

La percepción, aunque sucede por debajo del nivel de conciencia del receptor, se logra gracias a "una combinación de procesos cognoscitivos, unos comienzan a elaborar el código sensorial y otros que son inferencias y parten de nuestros conocimientos del mundo" (Best, como se citó en Vergara, 2012, p. 15).

En un proceso perceptivo se interpreta el estímulo según el nivel de comprensión de cada uno, realizando una adaptación de las tres fases propias de éste, que son: selección, organización e interpretación; en la primera fase el individuo realiza una percepción selectiva de un estímulo frente a los demás que se le presentan, dependiendo de sus intereses y necesidades; en la organización, la persona clasifica el estímulo, dándole un significado y resaltando aquellas características que son importantes para él; mientras que la interpretación, se apoya directamente en la "experiencia previa del individuo, así como de sus motivaciones, intereses personales y su interacción con otras personas... Así, la formación de los estereotipos corresponde en gran parte a la interpretación perceptual que el individuo da a los acontecimientos" (Guardiola, 2014, p. 13).

En este trabajo, la percepción sobre la que se indaga incluye las posibles amenazas, ventajas, limitantes y usos de artefactos tecnológicos en el aula de preescolar como herramienta didáctica que apoye el proceso de enseñanza, motive al aprendizaje de diversos contenidos y fortalezca las dimensiones del desarrollo. Por tanto, se entiende la percepción como "una imagen mental que se forma con ayuda de la experiencia y necesidades, después de un proceso de selección, organización e interpretación de las sensaciones" (Vergara, 2012, p. 17); así, la experiencia previa que hayan tenido los profesores en formación con el uso de cualquier artefacto tecnológico influye en su percepción, pues "toda percepción comporta una interpretación; y así, más que un fenómeno simplemente sensorial, es una conducta psicológica compleja que está vinculada a un cuadro de referencias particular, elaborado a partir de la propia experiencia personal y social" (Vergara, 2012, p. 15). 


\section{Método}

La investigación se realizó bajo un enfoque cualitativo con alcance descriptivo y diseño transeccional que incluye recolección de datos cuantitativos. La población objeto del estudio estuvo compuesta por 175 estudiantes de Pedagogía infantil de una universidad privada de la ciudad de Bogotá (Colombia). De esta muestra, a través de un muestreo aleatorio estratificado se seleccionó un grupo de 100 estudiantes en diferentes niveles de avance de sus estudios.

La pregunta que originó la investigación fue: ¿Cuáles son las percepciones que tienen los profesores en formación frente a las posibilidades didácticas de la inclusión de tecnología en el aula de educación inicial?

Como técnica de recolección de datos se utilizó la encuesta, para lo cual se diseñó e implementó un cuestionario con 20 ítems distribuidos en 15 preguntas tipo Likert con 5 opciones de respuesta, 3 de jerarquización y 2 preguntas de respuesta abierta, las cuales se diseñaron atendiendo 4 categorías iniciales de análisis (ver tabla I).

Tabla I. Categorías de análisis

\begin{tabular}{l|l}
\hline Categoría & Descripción \\
\hline Uso & Posibles usos que los profesores en formación darían a la tecnología. \\
Beneficios & Posibles ventajas o beneficios que los profesores perciben se obtienen con el uso de la tecnología. \\
Limitantes & Dificultades u obstáculos para el ejercicio de su práctica, asociadas con el uso de tecnología. \\
\hline Amenazas & Riesgo o desafíos que refieren los docentes al momento de implementar la tecnología. \\
\hline
\end{tabular}

Fuente: Elaboración propia.

Se realizó un análisis descriptivo de los datos recolectados a través del cuestionario generando histogramas y descriptores estadísticos de cada uno de los ítems. Con el propósito de comprender y ahondar en el sentido que los profesores en formación dieron a las respuestas del cuestionario se realizó un grupo focal con 9 estudiantes de la muestra, donde se analizaron las respuestas dadas por el grupo a las preguntas del cuestionario.

\section{Resultados}

Рara indagar sobre las percepciones de los docentes se diseñaron 4 preguntas relacionadas con tecnología en general (ver tabla II); el análisis de las respuestas dadas a estas cuatro preguntas permitió identificar qué elementos tecnológicos son considerados por los docentes en formación, como los más importantes, la necesidad de su uso en el aula, su rol como facilitador del aprendizaje y su autopercepción de habilidades tecnológicas. A continuación, se exponen los resultados detallados de las respuestas dadas por el grupo de estudiantes. 
Tabla II. Preguntas referidas a tecnología

1) Enumere de 1 a 9 la importancia de los elementos tecnológicos en su aula de clase teniendo en cuenta que 1 es menos importante y 9 más importante.

\begin{tabular}{|c|c|c|c|}
\hline Computadora & ( Tablets & (I Videojuegos & ( Teléfonos celulares \\
\hline Robots & ( grabadoras & ( Tableros digitales & ( Video beam \\
\hline
\end{tabular}

De acuerdo con las siguientes afirmaciones seleccione un punto de vista donde:

1. Totalmente en desacuerdo

2. En desacuerdo

3. Ni de acuerdo ni en desacuerdo

4. De acuerdo

5. Totalmente de acuerdo

\begin{tabular}{|c|c|c|c|c|}
\hline Afirmaciones & 1 & 2 & 3 & 4 \\
\hline 3) El uso de tecnología facilita los procesos de aprendizaje & & & & \\
\hline 4) Necesito tecnología para trabajar en mi aula de clase & & & & \\
\hline 5) Tengo formación necesaria para implementar la tecnología en el aula & & & & \\
\hline
\end{tabular}

Fuente: Elaboración propia.

\subsection{Percepciones asociadas a la relevancia de algunos artefactos tecnológicos en el aula}

Se investigó cuál es el elemento tecnológico que los estudiantes de licenciatura consideran más importante como apoyo en el desarrollo de su futura práctica docente y las razones por la cuales consideran su importancia. La pregunta utilizada fue de jerarquización, se solicitó al encuestado que asignara un valor de 9 a la herramienta que consideraba más importante, 8 a la siguiente en importancia y así hasta llegar a un puntaje de 1 a la que consideraba menos importante. En la tabla III se presentan los resultados promedio y las desviaciones de los puntajes asignados a cada uno de los elementos tecnológicos presentados.

Tabla III. Resumen estadístico de importancia de elementos tecnológicos que pueden utilizarse en el aula

\begin{tabular}{|c|c|c|c|c|c|c|c|c|c|}
\hline & 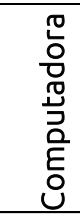 & 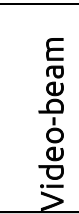 & 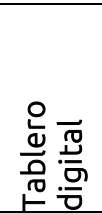 & $\frac{\stackrel{n}{0}}{\stackrel{.}{d}}$ & $\begin{array}{l}0 \\
0 \\
0 \\
0 \\
0 \\
0\end{array}$ & $\begin{array}{l}\frac{\omega}{0} \\
\frac{0}{0}\end{array}$ & $\begin{array}{l}\text { 으 } \\
\text { مे } \\
\text { ㅇ }\end{array}$ & 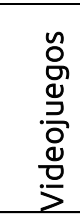 & $\frac{\frac{1}{3}}{\stackrel{J}{J}}$ \\
\hline Promedio & 7.30 & 6.50 & 5.80 & 5.50 & 5.40 & 4.90 & 4.20 & 3.60 & 3.00 \\
\hline Desviación estándar & 1.98 & 2.46 & 2.43 & 2.24 & 2.62 & 2.39 & 2.63 & 2.37 & 2.37 \\
\hline
\end{tabular}

Fuente: Elaboración propia.

Los estudiantes encuestados consideran que la computadora, el video beam (proyector) y los tableros digitales son las herramientas a las que más uso se les puede dar en un aula. En el grupo focal las principales razones sobre la preferencia del computador como la herramienta más importante en el aula se vieron ligadas a tres aspectos: 1) las computadoras son recursos de fácil acceso, "actualmente en todos lados vas a tener una" (estudiante de 7o. semestre); los estudiantes entrevistados perciben que la computadora es una herramienta "versátil y donde se adquiere mucha información" (estudiante 50. semestre); finalmente, perciben que es importante al convertirse en una herramienta de uso común en diferentes aspectos de la vida diaria y por las facilidades que ofrece para buscar información, comunicarse y elaborar diferentes tipos de trabajo (presentaciones, textos, etc.), "todo el mundo tiene un computador y sabe utilizarlo porque es necesario para la universidad y para todo" (estudiante 7o. semestre).

Los otros elementos que siguieron en importancia son el video beam y el tablero digital. Durante el grupo focal se puede evidenciar que los estudiantes ven en la proyección de contenidos un uso central de la tecnología en el aula. Dicha postura es reforzada por el ambiente de clase en el que son formados los futuros licenciados, en el que tanto el computador como el video beam son los medios tecnológicos más utilizados por sus profesores en la universidad. 
Según los resultados de la encuesta, los elementos tecnológicos con menor posibilidad de uso en el aula de educación inicial son los teléfonos celulares y los videojuegos. El $65 \%$ de los encuestados asignó al teléfono celular una puntación igual o inferior a 3 ( $46 \%$ de los encuestados asignaron un puntaje de 1) de igual forma el 64\% de los encuestados asignó una calificación igual o menor a 3 a los videojuegos (51\% de los encuestados asignó un valor de 2 y 3 ).

Las respuestas dadas por los estudiantes en los grupos focales muestran posiciones encontradas, por una parte hay percepciones negativas frente al uso de los celulares en el aula, pues consideran que "no tienen por qué estar en el aula y mucho menos con niños de esa edad" (estudiante de 3er. semestre), "los teléfonos celulares generan adicción y dependencia" (estudiante de 4o. semestre). Otros estudiantes opinan que pueden usarse con la mediación efectiva del profesor y proponiendo el uso de aplicaciones novedosas; sin embargo, atribuyen su poco o nulo uso al desconocimiento de aplicaciones apropiadas y estrategias para su gestión.

Algo similar se puede observar con relación a los videojuegos. Durante los grupos focales, en su mayoría, los participantes estuvieron en desacuerdo con la clasificación en un nivel bajo de importancia de los videojuegos, consideran que existen muchos que pueden ayudar y ser motivantes para los estudiantes "conozco muchos videojuegos que pueden ayudar al aprendizaje de los niños" afirma estudiante de 60. semestre. Algunos estudiantes tienen una percepción negativa de su uso porque lo consideran un distractor, "los niños pierden el tiempo en esos juegos y son muy adictivos" (estudiante 2o. semestre). El desconocimiento sobre el uso de esta herramienta en el aula puede ser el principal influyente al tomar la decisión de qué materiales utilizar, "creo que hay desconocimiento de estos objetos con fines educativos" señaló un estudiante de 80. semestre.

\subsection{Percepción sobre la necesidad de tecnología en el aula}

La encuesta muestra una división de opiniones frente a la afirmación "Necesito tecnología para trabajar en el aula" (ver figura 1). El 39\% de los encuestados manifestó estar de acuerdo con la afirmación, mientras un $27 \%$ estuvo en desacuerdo. Los demás participantes optaron por una postura neutral.

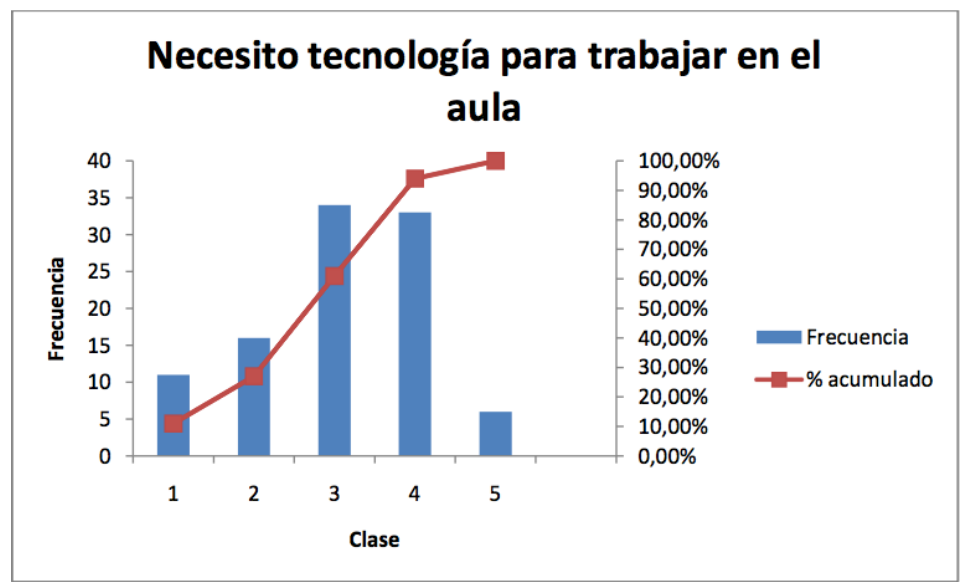

Figura 1. Histograma de respuestas asociadas con la aceptación de la afirmación "Necesito tecnología para trabajar en el aula"

La anterior división de opinión se manifiesta, igualmente, durante los grupos focales. Se evidenció que los estudiantes de 20., 40. y 70. semestre, no consideran necesaria la tecnología para trabajar en el aula, argumentan que por muchos años no se ha usado y el aprendizaje ha funcionado: "No lo veo necesario si está en uso otro material" (estudiante 20. semestre), existen otros materiales y herramientas que pueden utilizarse en el aula y no son tecnológicas. Por otra parte, las estudiantes que estuvieron de acuerdo con la necesidad de la tecnología saben que puede ser muy motivante y llamativa: "La sociedad y los avances tecnológicos hacen que sea necesario en el aula, pero no en exceso" (estudiante de 9o. semestre). 


\subsection{Percepción de la tecnología como facilitador del aprendizaje}

El 79\% de los encuestados estuvo de acuerdo o totalmente de acuerdo frente a la afirmación "La tecnología facilita el aprendizaje" (figura 2), un 7\% manifestó estar en desacuerdo.

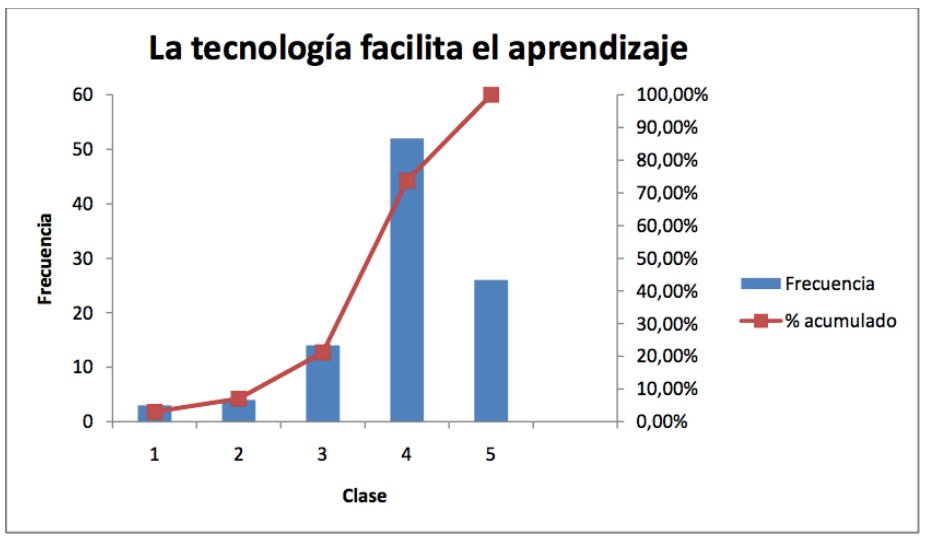

Figura 2. Histograma de respuestas asociadas con la afirmación "La tecnología facilita el aprendizaje"

Durante la entrevista en los grupos focales, los docentes en formación manifiestan que la tecnología facilita el aprendizaje porque hay una conexión directa entre los niños y la tecnología: "Los niños son nativos digitales y siempre les llamará la atención la tecnología" (estudiante 5o. semestre), por el interés y la motivación que genera en los estudiantes, porque es más llamativo para todos; "la motivación es clave para el aprendizaje y estas herramientas permiten eso", afirmó un estudiante de 7o. semestre. En esta pregunta todos estuvieron de acuerdo con que sí facilitan el aprendizaje: "También este aprendizaje puede deberse al uso que el docente le dé" (estudiante 8o. semestre).

\subsection{Percepción sobre la formación tecnológica de los futuros profesores}

Frente a la afirmación "Tengo la formación necesaria para usar tecnología en mis clases" (figura 3), el 45\% de los encuestados manifestó estar de acuerdo y un $28 \%$ en desacuerdo.

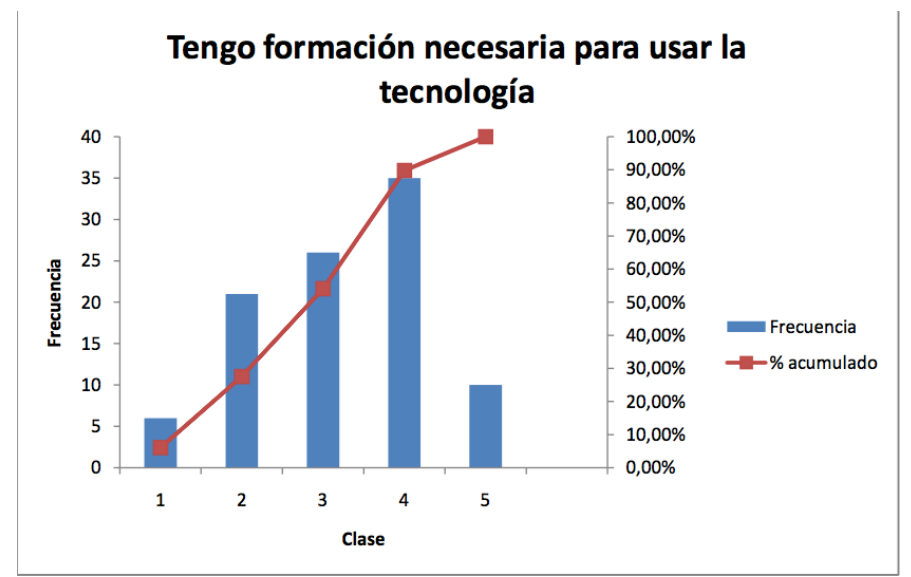

Figura 3. Histograma de respuestas asociadas con la afirmación

"Tengo la formación necesaria para usar la tecnología"

Esta pregunta generó interrogantes en el grupo focal, teniendo en cuenta que no sabían en qué consistía tener la formación necesaria, la mayoría de los docentes en formación (46\%) afirmó que tienen las capacidades básicas para trabajar con tecnología en el aula (Power Point, Prezi, edición de videos): "Sé manejar lo básico y con ello me puedo defender en el aula" (estudiante 7o. semestre), pero al mismo tiempo, estudiantes de 80. y 9o. semestre dijeron que no tenían la formación y consideran que en la universidad no hay muchas materias que se enfoquen a la enseñanza con tecnología: "Creo que no estoy capacitada para los niños de hoy en día y que necesitaría aprender más de tecnología", afirmó una estudiante de 80 . semestre. 


\section{Conclusiones}

Aunque estos estudiantes forman parte de la generación millenial y son nativos digitales, al reflexionar sobre su práctica profesional no ven un uso potencial de la tecnología en el aula como herramienta didáctica que apoye los procesos de enseñanza, afirmación apoyada por Sánchez-Labella (2015) en cuanto al uso enfocado únicamente al entretenimiento.

En los docentes en formación se evidencia una percepción tradicional del uso de la tecnología en el aula, relacionada con la falta de procesos formativos en esta área. Esta postura muestra cómo se intenta sustituir la escritura en el tablero con presentaciones preparadas previamente, en las cuales se puede exponer el contenido (apoyado en algunos casos por imágenes y videos), limitando el uso de la tecnología en el aula a un elemento de presentación de contenidos. Por lo anterior se infiere que los docentes en formación se encuentran en un nivel básico del uso de tecnología en el ámbito educativo (UNESCO, 2008).

Los docentes en formación no consideran otros elementos tecnológicos como recursos adecuados para el desarrollo de sus actividades pedagógicas, por el contrario, elementos como videojuegos y celulares se convierten en obstáculos o distractores de la enseñanza. Por lo anterior, se hace evidente la necesidad de capacitar en procesos de enseñanza adecuados, como lo sugieren Roblizo y Cózar (2015).

La mayoría de futuros profesores ven el uso de la tecnología como un elemento que motiva y facilita el aprendizaje, pero no expresan la importancia de incluirla en la clase, por ello la inclusión de espacios para el aprendizaje, apropiación y reflexión sobre el uso didáctico de la tecnología se trasforma en una necesidad de formación actual para los futuros docentes.

La contribución de manera transversal al desarrollo de competencias tecnológicas y profesionales de los futuros educadores infantiles -que les permitan hacer usos efectivos y eficientes de los recursos tecnológicos existentes y venideros en sus aulas- se convierte en un elemento adecuado de formación.

\section{Referencias}

Burin, D., Coccimiglio, Y., González, F. y Bulla, J. (2016). Desarrollos recientes sobre habilidades digitales y comprensión lectora en entornos digitales. Psicología, Conocimiento y Sociedad, 6(1), 191-206.

Bustos, J. S., Aguas, J. R., Díaz, C. C. y Roa-Guerrero, E. E. (2017). Diseño e implementación de una plataforma robótica móvil para identificación de minas terrestres antipersona en diferentes terrenos del territorio colombiano. TECNIA, 25(1), 1-10.

Cabero, J. (2016). ¿Qué debemos aprender de las pasadas investigaciones en Tecnología Educativa? Revista Interuniversitaria de Investigación en Tecnología Educativa, 0, 23-33. doi:10.6018/riite/2016/256741

DANE. (2017). Indicadores básicos de tenencia y uso de Tecnologías de la Información y la Comunicación -en hogares y personas. Recuperado de https://www.dane.gov.co/files/investigaciones/boletines/tic/prese tic hogares 2016.pdf

Carbajo, N. L. y Carcelén, C. Q. (2016). Creativiza-T: la creatividad a su disposición. Sevilla, España: Punto Rojo Libros.

Dorfsman, M. I. (2012). La profesión docente en contextos de cambio: el docente global en la sociedad de la información. Revista de Educación a Distancia - Docencia Universitaria en la Sociedad del Conocimiento, 6, 1-23. Recuperado de https://www.um.es/ead/reddusc/6/marcelo dusc6.pdf

Drigas, A., Kokkalia, G. y Lytras, M. D. (Octubre de 2015). ICT and collaborative co-learning in preschool children who face memory difficulties. Computers in Human Behavior, 51, 645-651.

Druin, A. y Hendler, J. (2000). Robots for kids: exploring new technologies for learning. EUA: Morgan Kaufman Publishers.

Escofet, A., López M. y Alvarez, G. (2014). Una mirada crítica sobre los nativos digitales: análisis de los usos formales de TIC entre estudiantes universitarios. Revista Q, 9, 1-19.

Fernández-Cruz, F. J. y Fernández-Díaz, M. J. (2016). Los docentes de la Generación Z y sus competencias digitales. Comunicar, Revista Científica de Educomunicación, 24(46), 97-105. 
Galvis, Á. (2004). Oportunidades educativas de las TIC. Recuperado de

http://www.colombiaaprende.edu.co/html/investigadores/1609/articles-73523 archivo.pdf

Guacaneme-Mahecha, M., Gómez-Zermeño, M. G. y Zambrano-Izquierdo, D. (2016). Apropiación tecnológica de los profesores: el uso de recursos educativos abiertos. Educación y Educadores, 19(1), 105117. doi:10.5294/edu.2016.19.1.6

Guardiola P. (2014). Mis publicaciones de sociología. Recuperado de http://www.um.es/docencia/pquardio/documentos/percepcion.pdf

TED. (2011, junio 10). Scott Hess - Millennials: who they are y why we hate them [video]. Recuperado de https://ed.ted.com/on/mVZXkrQd

Kashiwagi, M., Liu, L., Chu, K. K., Sun, C-H, Tanaka, A., Gardecki J. A. y Tearney, G. J. (2014) Feasibility of the assessment of cholesterol crystals in human macrophages using micro optical coherence tomography. PLOS ONE, 9(7): e102669. doi:10.1371/journal.pone.0102669

Lindahl, M. G. y Folkesson, A. M. (2012). ICT in preschool: friend or foe? The significance of norms in changing practice. International Journal of Early Years Education, 20(4), 422-437.

Litwin, E. (2005). Tecnologías educativas en tiempos de Internet. Buenos Aires: Amorrortu.

Liu, X., Toki, E. I. y Pange, J. (2014). The use of ICT in preschool education in Greece and China: a comparative study. Procedia - Social and Behavioral Sciences, 112, 1167-1176. Recuperado de https://www.sciencedirect.com/science/article/pii/S1877042814012981

Marques, P. (2013). Impacto de las TIC en la educación. Funciones y limitaciones. 3C TIC, 2(1), 1-15.

McAuliffe, M. (2016). The potential benefits of divergent thinking and metacognitive skills in STEAM learning: a discussion paper. International Journal of Innovation, Creativity and Change, 2(3), 71-82.

Morales, M., Trujillo, J. M. y Raso, F. (2015). Percepciones de la integración de las TIC en el proceso de enseñanza-aprendizaje de la Universidad. Píxel-Bit. Revista de Medios y Educación, 46.

doi:10.12795/pixelbit.2015.i46.07

Morduchowicz, R. (2012). Los adolescentes y las redes sociales. Buenos Aires: Fondo de Cultura Económica.

Mulligan, H., Rowland, J. L., Sandlund, M., Potterton, J. y Kanagasabai, P. (2015). Emerging use of interactive technology in rehabilitation for young people. Physiotherapy, 101, e16-e17.

Mundy, M. A., Kupczynski, L. y Kee, R. (2012). Teacher's perceptions of technology use in the schools. SAGE Open, 2(1).

Neisser, U. (1981). Cognición y realidad: principios e implicaciones de la psicología cognitiva. Alemania: Marova.

Núñez, L., Conde, S., Ávila, J. y Mirabent, M. (2015). Implicaciones, uso y resultados de las TIC en educación primaria. Estudio cualitativo de un caso. Edutec. Revista Electrónica de Tecnología Educativa, 53, 1-17. doi:10.21556/edutec.2015.53.581

OECD. (2002, abril 25-26.). Reviewing the ICT sector definition: Issues for discussion, Working party on indicators for the information society. Estocolmo.

Ok, M., Cho, H. R. y Cho, Y. H. (2016). Instructional support for peer assessment in STEAM design education. Ponencia presentada en la Conferencia de la Sociedad Coreana de Tecnología Educativa. Lugar? Recuperado de https://m.happycampus.com/paper-doc/16620266/\#detail

Ortiz, G. (2011). L@s jóvenes y su relación con la red Interner: de la adicción al consumo cultural. Razón y palabra, 78.

Piscitelli, A. (2009). Nativos digitales. Buenos Aires: Santillana.

Pozuelo, J. (2014). ¿Y si enseñamos de otra manera?: competencias digitales para el cambio metodológico. Caracciolos: Revista digital de investigación en docencia, 2 (1), 1-21.

Prensky, M. (2001). Nativos e inmigrantes digitales. On the Horizon, 9(5), 1-6. 
Roblizo, M. y Cózar, R. (2015). Usos y competencias en tic en los futuros maestros de educación infantil y primaria: hacia una alfabetización real para docentes. PíXel-Bit. Revista de Medios y Educación, 0(47), 2339. doi:10.12795/pixelbit

Sánchez-Labella, Martín I. (2015). Las tablets como herramientas educativas para la infancia. Neox Kidz, un estudio de caso. Redes.com: revista de estudios para el desarrollo social de la Comunicación, 12, 47-67.

Shatto B., Erwin K. (2016). Moving on from millennials: preparing for Generation Z. Journal of Continuing Education in Nursing, 47(6), 253-254. doi:10.3928/00220124-20160518-05

Tello, I. y Cascales, A. (2015). Las TIC y las necesidades específicas de apoyo educativo: análisis de las competencias TIC en los docentes. Revista Iberoamericana de Educación a Distancia, 18(2), 355-383.

doi:10.5944/ried.18.2.13536

Turing, A. (1950). Computing machinery and intelligence. Mind, 59(236), 433-460. Recuperado de http://www.jstor.org/stable/pdf/2251299.pdf?refreqid=excelsior\%3A30397222fd723320cd5be4de8015 $\underline{\mathrm{e} 9 \mathrm{a} 7}$

UNESCO. (2008). Estándares de competencias en TIC para docentes. Recuperado de www.oei.es/historico/tic/UNESCOEstandaresDocentes.pdf

Vergara, M. J. (2012). Percepción de docentes y estudiantes sobre el currículo de estudios de una universidad privada de Lima. Tesis para optar al grado de Maestro en Educación. Recuperado de http://repositorio.usil.edu.pe/bitstream/123456789/1348/1/2012 Vergara Percepci\%C3\%B3n-de-

docentes-y-estudiantes-sobre-el-curr\%C3\%ADculo-de-estudios-de-una-universidad-privada-de-Lima.pdf 